\title{
Inclusions in melting process of titanium and titanium alloys
}

\author{
Meng-jiang Cen ${ }^{1}$, *Yuan Liu ${ }^{1,2}$, Xiang Chen ${ }^{1,2}$, Hua-wei Zhang ${ }^{1,2}$, and Yan-xiang Li ${ }^{1,2}$ \\ 1. Department of Materials Engineering, School of Materials Science and Engineering, Tsinghua University, Beijing 100084, China \\ 2. Key Laboratory for Advanced Materials Processing Technology (MOE), Tsinghua University, Beijing 100084, China
}

\begin{abstract}
This paper summarizes melting methods of titanium and titanium alloy, such as vacuum arc melting (VAR) and electron beam cold hearth melting (EBCHM), and the related inclusions formed when using these melting methods. Low-density inclusions are resulted from contamination of air, and high-density inclusions are caused by refractory elements. The formation process of inclusions was analysed. The removal mechanism of different kinds of inclusions was specified. Low-density inclusions are removed mainly by resolving. This is a comprehensive process containing reaction diffusion. The resolving rate of high-density inclusions is so low that these inclusions are mainly removed by sedimentation. The experiments and physical models of inclusions are detailed. In various melting methods, vacuum arc melting is prominent. However, this method cannot remove inclusions effectively, which usually results in repeat melting. Electron beam cold hearth melting has the best ability of removing inclusions. These results can provide instructions to researchers of titanium and titanium alloys.
\end{abstract}

Key words: titanium; inclusions; vacuum arc melting; electron beam cold hearth melting

CLC numbers: TG146.23 Document code: A Article ID: 1672-6421/2019)04-223-09

$\mathrm{T}$ itanium and its alloys have excellent properties such as light weight, corrosion resistance and high temperature mechanical strength. The applications in ship, marine, aerospace and civil products are increasing rapidly ${ }^{[1-7]}$. For example, the fraction of titanium parts of SU-27, F-14 and F-15 fighter jets is $15 \%, 24 \%$ and $27 \%$, respectively. The consumption of titanium of F-22 jet reaches $41 \%{ }^{[8-9]}$. At present, the melting methods of titanium mainly include vacuum arc melting (VAR), electron beam cold hearth melting (EBCHM), vacuum induction melting (VIM) and plasma arc meting $(\mathrm{PAM})^{[10-15]}$. The raw materials of titanium have broad sources, and titanium is so active in the melt state that it may be oxidized or nitrided. So, inclusions may be introduced in the production process.

Inclusions can deteriorate the mechanical properties of titanium parts (especially for rotating parts) ${ }^{[16]}$. For example, the mechanical property of turbine blades depends on the size and number density of inclusions. Several disasters in aviation history are related to metallurgical defects of titanium parts. This can be seen

\section{*Yuan Liu}

Male, born in 1974, Ph.D., Associate Professor. His research interests mainly focus on porous materials and solidification theory.

E-mail: yuanliu@mail.tsinghua.edu.cn

Received: 2019-04-04; Accepted: 2019-05-09 from the report of the Federal Aviation Administration. These defects are mainly hard $\alpha$ inclusions ${ }^{[17]}$. To promote the quality of titanium parts used for aerospace and meet the need of advanced materials in aeroengines, many countries are paying more attention in recent years to the preparation and processing technology of titanium alloys.

\section{Melting methods of titanium and titanium alloys}

In the melt state, titanium is active and tends to react with nitrogen and oxygen, so the melting process needs to proceed in a vacuum. Since the middle twentieth century, the melting method of titanium has been mainly vacuum arc melting (VAR). In the low pressure or inert gas atmosphere, the arc produced between the electrode and the crucible is used as the heating source. The electrode of consumable raw material is melted and dropped into the crucible to become an ingot. The merits of VAR include the cost of equipment and maintenance is relatively low, the operation of equipment is easy, and the composition of ingots is homogeneous. However, this melting method has inherent disadvantages which usually leads to serious quality problems (especially for high-density inclusions) if the technology is not 
appropriate. These defects have a higher density and melting point than titanium, and a lower resolving rate. So eventually, they deposit into the bottom of the melt. These inclusions are highly incompatible with the alloy matrix. Therefore, cracks may occur in the subsequent processes. These cracks are fatal for the performance of titanium parts. There are specific requirements on how to guarantee the homogeneity of composition, mixing the sponge intermediate alloy, compressing the raw material, and combining different sections by welding to produce the electrode with a high strength. Normal ingots need twice re-melting, while ingots used for rotating parts need thrice re-melting ${ }^{[18]}$. In some circumstances, the most severe metallurgical defects of the highand low-density inclusions cannot be totally eliminated even by thrice re-melting, to which the failure and fracture of most titanium parts are directly related ${ }^{[19]}$.

In the late eighties, electron beam cold hearth meting (EBCHM) technology was introduced to promote the metallurgical quality of titanium and their alloys, and improve the quality of titanium used for aviation. The main characteristic of EBCHM is the application of cold hearth technology. The power of the electron gun, scan frequency and residual time of the electron beam on the melt surface can be easily manipulated in the melting process. The electron beam has a very high energy density $\left(10^{3}-10^{6} \mathrm{~W} \cdot \mathrm{cm}^{-2}\right)$, so, the temperature of molten metal can be very high. The melting process is proceeded in the vacuum atmosphere $\left(<10^{-3} \mathrm{~Pa}\right)$, so toxic impurities and gases can be effectively removed. The EBCHM is suitable for the melting of metals which is active in the melt state (Ti, Zr, V, Hf), refractory metals (W, Mo, Ta, Nb), and their alloys ${ }^{[20]}$. In the melting process, raw materials are melted in the feeding port and then flow into the crucible. When molten metal flows through the refining cold hearth, high density inclusions (HDI) drop into the bottom of the melt and are removed permanently as the effect of gravity. Low density inclusions (LDI) have the advantageous condition to be effectively removed by floating, evaporation and resolving. Therefore, if the melting parameters are appropriate, the EBCHM can produce high quality titanium ingots without segregation and inclusions by the single melting process ${ }^{[21-26]}$. Meanwhile, the CBCHM can use recycled titanium as raw material, which is economical ${ }^{[27]}$. In addtion, plasma beam cold hearth melting has a similar principle with the EBCHM, with the distinction of using different heating sources. For induction melting, the ingot size is limited.

At present, producing high quality titanium ingots using the EBCHM or EBCHM and VAR coupling melting has become a tendency ${ }^{[28]}$. The objects of concern are the homogeneity of composition, the removal of inclusions and the evaporation of volatile elements. Melting parameters have a great influence on these processes. However, the removal of inclusions and evaporation are usually a contradiction. In the production process of titanium using the EBCHM, reasonable parameters are very important to realize optimal inclusion removal and minimum evaporation loss ${ }^{[29]}$.

In general, the VAR is incompetent for the inclusion removal, while the EBCHM and plasma arc melting have a very good effect. However, the LDI may still exist in ingots prepared by coupled the EBCHM and VAR. Multiple re-melting by the VAR results in inhomogeneity, and the inhomogeneity is more obvious for the EBCHM. The EBCHM is suitable for melting of titanium and titanium alloys with a low content of alloying elements, because evaporation is severe in the EBCHM process. Plasma arc melting is suitable for melting titanium alloy, while pores and surface segregation may exist in the ingots. Ingots prepared by the EBCHM have a cost advantage for rolling sheets, while this advantage may disappear for other kinds of products ${ }^{[30]}$.

\section{Low-density inclusions}

Low-density inclusions (LDI) are the solid solution or compounds of titanium and other light elements, which are resulted by contamination of nitrogen, oxygen or carbon. The Ti-N inclusions are the most harmful. This is because their resolving rate is very slow, so they remain in ingots or parts after the VAR melting. The density of LDI is close to that of titanium matrix, and their crystal structure is coherent to the matrix. These inclusions are difficult to detect by nondestructive testing methods. The LDI seriously deteriorate the fatigue resistant property of materials and become a potential risk. When these materials are used for rotating parts, such as compressor discs or turbines of aero-engines, Ti-N inclusions may cause the failure of the engine parts and result in an air crash. The effect of oxygen is similar with that of nitrogen. The distinction is the increasing tendency of melting point being lower when the concentration of oxygen increases. Oxygen has a higher diffusion coefficient than nitrogen in the $\beta$ phase, which facilitates the resolving of inclusions, so the addition of $\mathrm{TiO}$ to the melt will not result in $\mathrm{TiO}$ inclusions.

Intrinsically, Ti-N inclusions are $\alpha$ solid solution or $\delta$ compounds. It can be seen from Fig. 1 that titanium and nitrogen can form $\beta$ phase, $\alpha$ phase and $\delta$ phase with the increase of nitrogen. Within these inclusions, $\alpha$ phase is the most common in production. The mass fraction of nitrogen in the $\beta$ phase is lower than $1 \%$. For the $\delta$ TiN, the mass fraction of nitrogen reaches $47.4 \%$, whose theoretical density at room temperature is $5.39 \mathrm{~kg} \cdot \mathrm{m}^{-3}$. At $3,290{ }^{\circ} \mathrm{C}, \delta$ phase decomposes with the reaction: $\delta \mathrm{TiN} \rightarrow 1 / 2 \mathrm{~N}_{2}+\mathrm{Ti}$.

The microstructure of $\alpha$ inclusions is shown in Fig. 2. It can be seen that there are cracks and pores around the inclusion, which makes parts containing $\alpha$ inclusions apt to produce fatigue cracks. The actual density of $\alpha$ phase is higher than that of titanium matrix. However, these inclusions usually have porous structures, which makes the general density lower than that of the matrix. These inclusions have no specific definition of structure. The detected nitrogen concentration in the practical production is lower than $14 \%$. In the EBCHM process, these inclusions still have access to go into the crystallizer and eventually become defects. It is very difficult to detect this kind of inclusion by X-ray or supersonic method if no cracks reside around the inclusion, so it is crucial to remove these inclusions 


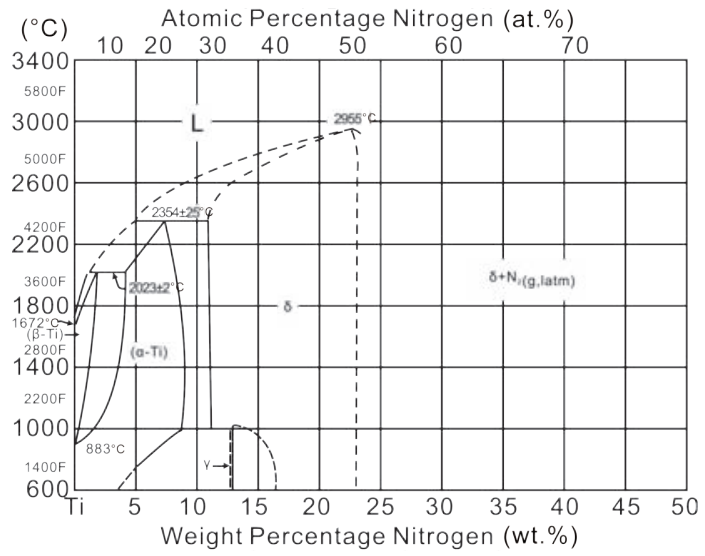

Fig. 1: Binary phase digram of $\mathrm{Ti}^{\mathrm{i}} \mathrm{N}^{[31]}$

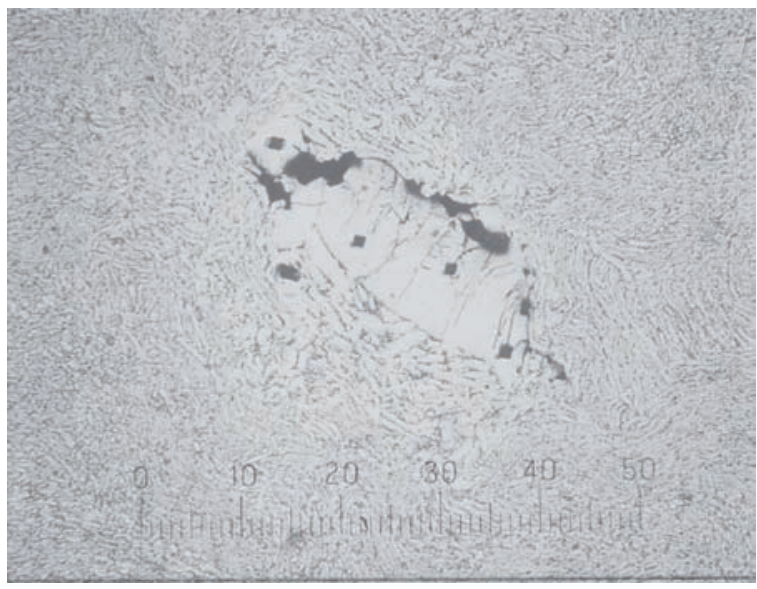

Fig. 2: Microstructure of $\alpha$ inclusion $^{[31]}$

in the melting stage. The recognition concerning $\alpha$ inclusions can be summarized as follows ${ }^{[31]}$ :

(1) $\alpha$ inclusions can be caused by nitrogen alone or by the combining effect of nitrogen and oxygen;

(2) Working parts containing $\alpha$ inclusions do not necessarily generate cracks, it dependson the working circumstance;

(3) $\alpha$ inclusions may emerge on any location of ingots for the VAR method.

The nitrogen contamination of titanium mainly occurs during the production and melting process of titanium sponge ${ }^{[32]}$. The production of titanium sponge usually contains a reduction reaction process, which proceeds on a sealed container. If the container is damaged, the titanium will contact with the air and be severely oxidized and nitrided. Ingots may be oxidized or nitrided during the VAR melting process. For example, if the seal is not firm or the vacuum degree is not sufficient when welding the compressed titanium electrode in the plasma welding machine or in the furnace. Although nitride inclusions in the titanium sponge exist in the form of fine particles, they can remain in the ingots in the VAR melting process or even in multiple melting processes. Henry et al. ${ }^{[33]}$ studied the possible sources of Ti-N inclusions of the titanium sponge produced by the magnesium reduction method. Their research simulated the air leak and the effect of air contamination of magnesium in the reduction and distillation process. The results show that the distribution of nitrogen in the prepared titanium sponge is greatly inhomogeneous. Some areas showed nitrogen concentration reaching $12 \%$, and these areas contain $\mathrm{Ti}_{2} \mathrm{~N}$, $\delta \mathrm{TiN}$ or both. If the contaminated titanium sponge is used as raw material, hard $\alpha$ inclusions can be detected in the ingots.

The experimental and the oretical research works of Ti-N inclusions are mainly concentrated in the 1980 s to the 1990 s. Bewlay et al. ${ }^{[34]}$ researched the resolving process of TiN ( $\delta$ phase) in the Ti-6242 melt. The results show that $\alpha$ phase and $\beta$ phase occur between the $\delta$ phase and liquid phase after a period of resolving. Some alloying elements won't diffuse into the $\delta$ phase. These elements are aluminum, zirconium, zinc and molybdenum. The nitrogen fraction in the $\alpha$ phase is relatively high. The $\beta$ phase contains aluminum, zirconium, zinc and molybdenum, while their concentration is lower than that of the melt.

Resolving is an important method of removing Ti-N inclusions, and the reported resolving rate of Ti-N inclusions is shown in Table 1 . The resolving process of Ti-N inclusions contains solid phase transformation. Figure 3 shows the microstructure of $\alpha$ inclusion in the resolving process, which clearly illustrates the $\beta$ phase and the transition layer between $\alpha$ and $\beta$ phase. Wang et al. ${ }^{[35]}$ studied the effect of alloying elements on the diffusion of nitrogen in the Ti/N interface. The chosen alloy system is $\mathrm{CpTi}$, Ti64, Ti17 and Ti6242, which contains various stabling elements of $\alpha$ and $\beta$ phase. In the VAR melting process, the titanium sponge particles were added to the melt, and the resolving process was studied. The results show that the alloying elements will retard the diffusion of nitrogen, especially when the melt contains stabilizing elements of $\alpha$ phase.

Bellot et al. ${ }^{[36]}$ studied the resolving of $\alpha$ inclusion in the pure titanium melt and established the resolving model. The experiment process is shown in Fig. 4, and the melting equipment is an electron furnace equipped with a $30 \mathrm{~kW}$ electron gun. The synthesized $\alpha$ particle was put into the melt, and then the resolving of particle was observed after some holding time. In this experiment, the sample was fixed at the tip of a tungsten bar. The bar was stretched into the melt which was scanned by electron beam. The depth of the sample in the melt and the holding time is constant.

The heating was stopped after some holding time, and then the material was cooled to room temperature. The microstructure and composition distribution of the particle

Table 1: Resolving rate of TiN inclusions ${ }^{[34]}$

\begin{tabular}{|c|c|c|c|}
\hline Authors & $\begin{array}{l}\text { Dissolving } \\
\text { species }\end{array}$ & Parent alloy & $\begin{array}{c}\text { Dissolution rate } \\
\left(\mu \mathrm{m} \cdot \mathrm{s}^{-1}\right)\end{array}$ \\
\hline Henry et al. & TiN & $\mathrm{Ti}$ & 3.7 \\
\hline Guillon et al. & TiN & Ti-64 & 0.37 \\
\hline Pannen et al. & TiN & Ti-64 & 1.6 \\
\hline $\begin{array}{l}\text { Buttrill and } \\
\text { Shamblen }\end{array}$ & TiN & $\mathrm{Ti}$ & 1.6 \\
\hline Bewlay & TiN & Ti-6242 & 2.2 \\
\hline
\end{tabular}


are shown in Fig. 5. It shows that $\beta$ phase forms on the particle surface after some holding time. According to the result of micro-analysis, the resolving process of $\alpha$ inclusion is controlled by the diffusion of nitrogen from the internal particle to the melt. After some holding time, the inclusion divided into two regions. The interior of the particles is homogeneous $\alpha$ phase which is the same as the initial state. At the outside of the particle, a layer of acicular $\beta$ phase forms. The thickness of $\beta$ layer is related to the resolving condition, and the layer is thicker in the static melt than that in the flowed melt.

According to the experiment result, Bellot established the resolving model of $\alpha$ inclusion, as shown in Fig. 6. This model describes the resolving process of a dense $\alpha$ inclusion in the titanium bath. The heating and cooling processes of inclusions

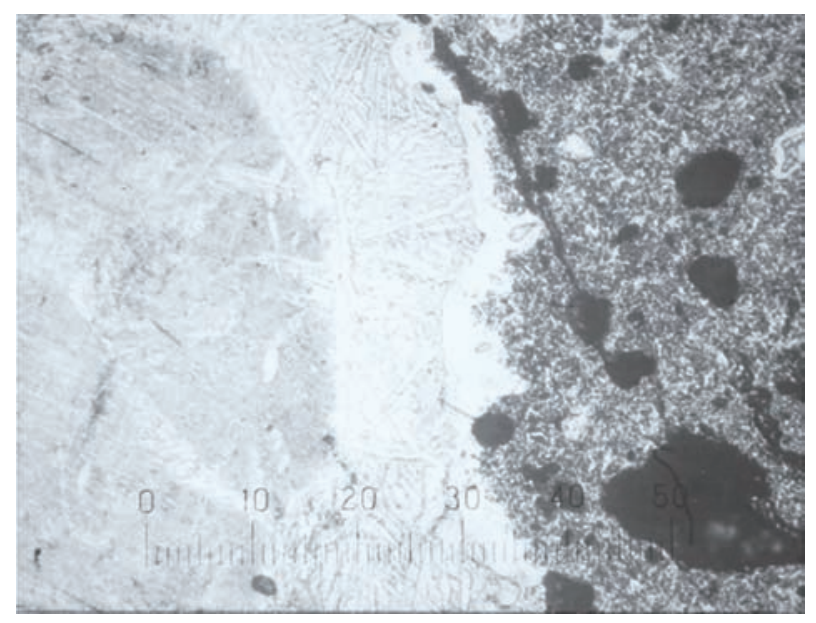

Fig. 3: Microstructure of partially resolved $\alpha$ inclusion ${ }^{[31]}$

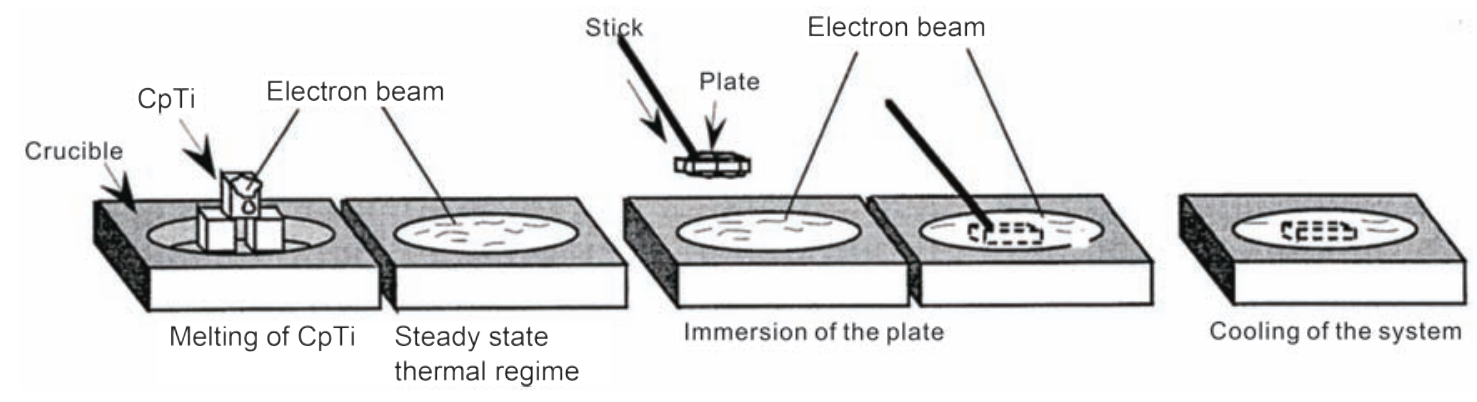

Fig. 4: Resolving experiment of $\alpha$ inclusion $^{[36]}$
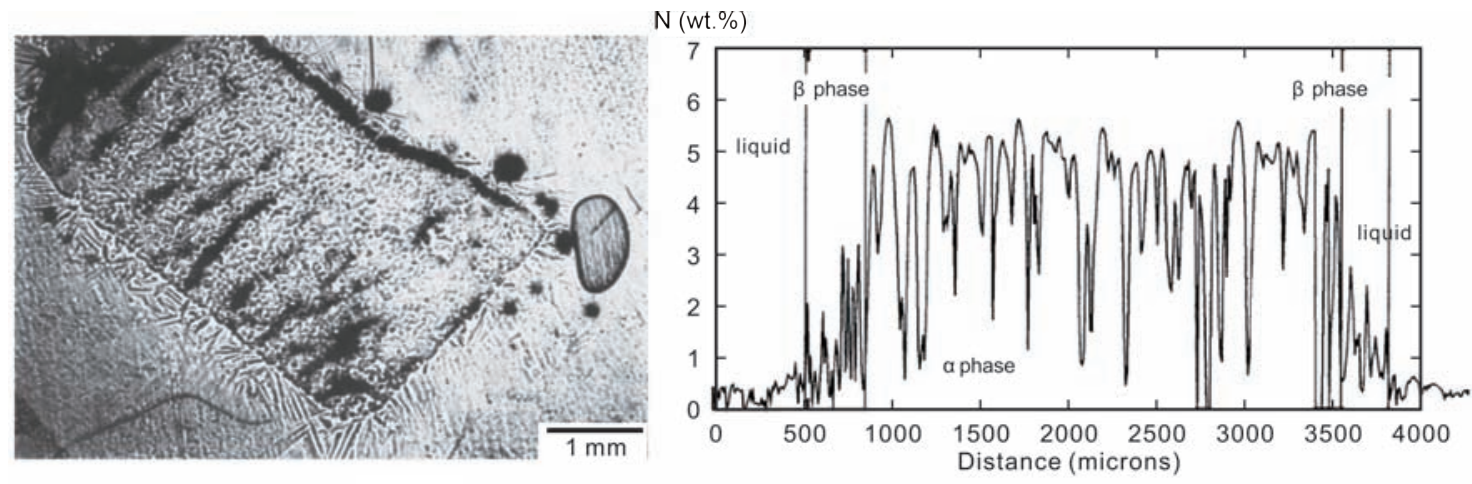

Fig. 5: Particle microstructure (left) and concentration distribution (right) ${ }^{[36]}$

as well as the micro-segregation which were formed in the solidification process are ignored. The model contains two regions, namely $\alpha$ and $\beta$ phase, and the size of inclusions and phases changes with the melting process. The following assumptions are made for simplification: the interface between two phases is in the state of thermodynamic equilibrium; the calculated region is one dimensional; the temperature and density of the inclusion are constant. The migration of interface at different time can be calculated according to Fick's law and mass conservation equation.

Figure 7 compared the calculating (solid line) and measurement (dotted line) result of nitrogen distribution in the $\alpha$ inclusion, and it illustrates that the calculated and experimental results are close with each other. The calculated result shows that a thick $\beta$ layer occurs on the inclusion surface at the initial stage of diffusion. From the calculated and measured results of the size of two phases, the calculated results of $\beta$ phase are more in line with the experiment compared to that of $\alpha$ phase.

The removing methods of $\alpha$ inclusions include diffusion annealing, high temperature decomposing and resolving. Diffusion annealing is expensive, time consuming and cannot completely remove inclusions. High temperature decomposing requires the melting temperature to exceed the melting point of $\alpha$ inclusion, which demands a high requirement for the heating source. Normal melting equipment, such as VAR, almost cannot realize high temperature decomposing as the melting temperature is far below the melting point of $\alpha$ inclusion. The 


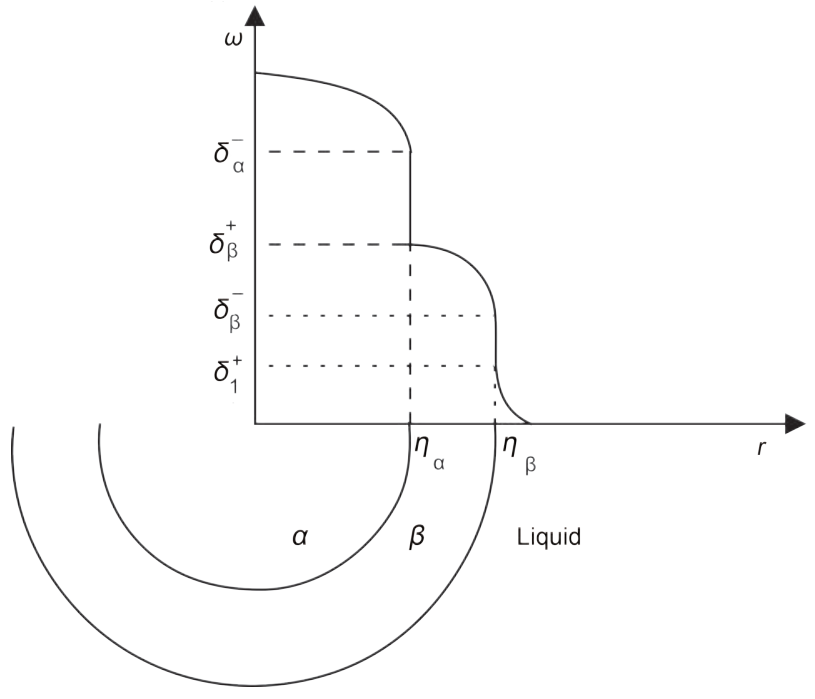

Fig. 6: Resolving model of $\alpha$ inclusion ${ }^{[36]}$

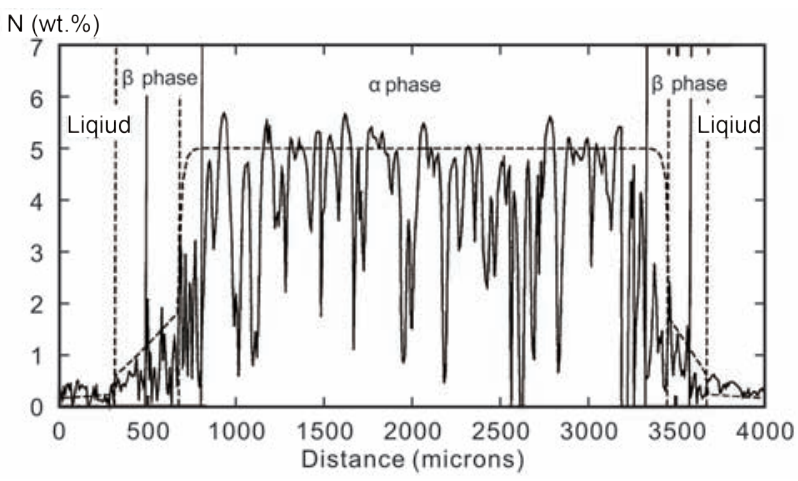

Fig. 7: Calculated (solid line) and measured (dotted line) results of nitrogen distribution in $\alpha$ phase ${ }^{[36]}$

resolving process proceeds by the diffusion of nitrogen at the melting temperature. This process requires certain residual time and dynamic conditions. Nitrogen needs to pass through the $\beta$ layer and this process is slow, which is the major obstacle of $\alpha$ inclusion removal.

\section{High-density inclusions}

High-density inclusions (HDI) are mainly resulted by high density refractory metal elements. The known HDI can be classified into two categories: refractory metal elements (W, $\mathrm{Mo}, \mathrm{Ta}, \mathrm{Nb}$ etc.) and their compounds (WC, TiW etc.). The physical properties of some common HDI and titanium are shown in Table 2, which shows that the melting point and density of HDI are higher than that of titanium. The shape and size of these particles have a great influence on the fatigue resistance of titanium parts. The sharper and larger the particles, the lower the fatigue resistance of parts.

Wang et al. ${ }^{[37]}$ analyzed the sources of tungsten inclusions in the titanium ingots produced by VAR and thought that there are two possible sources. The first is the added titanium wastes in the raw material containing debris from hard alloy machine tools which contain tungsten. Hard alloy debris can be
Table 2: Physical properties of some HDI and titanium ${ }^{[40]}$

\begin{tabular}{ccc|}
\hline Tungsten & $\begin{array}{c}\text { Melting point } \\
\left({ }^{\circ} \mathbf{C}\right)\end{array}$ & $\begin{array}{c}\text { Density } \\
\left(\mathbf{g} \cdot \mathbf{c m}^{-3}\right)\end{array}$ \\
\hline Molybdenum & 3,422 & 19.25 \\
Niobium & 2,623 & 10.28 \\
Titanium & 2,477 & 8.57 \\
& 1,668 & 4.5
\end{tabular}

eliminated by using magnetic selecting equipment or adding a manual selecting process for titanium wastes. Another source is the drop of tungsten electrode in the production process. Some methods can be taken to avoid this problem: replacing the tungsten electrode of plasma welding gun with a copper electrode, and cancelling the operation of manual plasma arc welding, which means all welding operations are proceeded in the welding chamber.

Some titanium alloys contain refractory elements. For example, $\beta$ titanium alloy became the focus in recent years for its superior formability and high strength. Also, the quality requirement of $\beta$ titanium is high. The $\beta$ titanium alloy contains stabilizing elements (V, Mo, Ta etc.) of $\beta$ phase with a weight fraction from several to dozens, and they are refractory elements. In the VAR process, these refractory particles are apt to drop to the melt bottom. Their density and melting point are high. These particles won't resolve completely in the melting process and will eventually become inclusions. In general, the major sources of HDI include ${ }^{[38-39]}$ :

(1) Contamination by debris of hard alloy machine tool, such as WC, when using titanium wastes as raw materials.

(2) Occasional drop into the melt from a filament, thermocouple et al. in the production process.

(3) Inappropriate preparation of raw material, such as adding refractory metal in a size too large to be removed by resolving.

Akihiro et al. ${ }^{[40]}$ researched the resolving of Ta, Mo and $\mathrm{V}$, which are refractory elements and alloying elements of $\beta$ titanium, in the single VAR melting process. Inclusion particles with different sizes were used. After some holding time, the change of particles is observed. From the experiment results, the following conclusions can be reached:

(1) Particles of Ta, Mo and V can be removed in the single VAR melting process when their diameter is less than 74, 149 and 3,360 $\mu \mathrm{m}$ respectively.

(2) V particles almost totally resolve in the melt of the electrode tip. However, Ta and Mo particles resolve mainly in the crucible.

(3) The time of total resolving of inclusions is exponential to the complexity of resolving.

(4) The size of particle has a critical value for complete resolving.

Li et al. ${ }^{[41]}$ studied the hard alloy inclusions of titanium alloys. The results show that these hard alloy particles just partially resolved after the melting. The edges of partially resolved 
particles are black in color and have obvious stages. The grain size of the particle interior is less than that of the surface. There is a transition boundary between the particles and the matrix. The boundary layer contains both matrix elements and inclusion elements, which means there is mutual diffusion between matrix elements and inclusion elements.

Ghaza et al. ${ }^{[42]}$ designed the resolving experiment of tungsten bar in the titanium melt, as shown in Fig. 8. The chosen titanium alloys are CpTi, Ti64 and Ti17, and the melting equipment is electron beam furnace. One kilogram of titanium was put into the water-cooled copper crucible. The refractory specimen was fixed on the tip of a stainless bar by tungsten wire. When the titanium was melted, the tungsten bar was stretched into the melt. After some holding time, fetch the specimen, and measure the size of the specimen. Two classes of experiments were carried out. In the first scenario, the specimen was tested after some holding time. In this experiment, the size of specimen can be accurately measured, so the resolving rate can be estimated. The other experiment is similar with the first one, the distinction is that the specimen was not removed from the melt after the melting and was cooled with the melt. This experiment can be used to research the diffusion of elements and the interaction of refractory elements with the melt.

According to the experiment result, Ghazal proposed the resolving model of refractory inclusions, as shown in Fig. 9. This model is based on the following assumptions: the temperature in the internal inclusion is homogeneous and it is the same with that of the melt; the solid/liquid phase interface is on the state of equilibrium, so the composition of the interface can be calculated according to phase diagram. The diffusion in the solid phase was neglected as the absence of diffusion coefficient of titanium and tungsten. This model may be considered reasonable as a first approximation. In fact, this resolving model is the simplification of a resolving model of hard $\alpha$ inclusion with the distinction that there is no solid phase transformation in the resolving process of HDI.

The melting temperature of titanium is lower than that of HDI, and the residual time of these inclusions in the melt is very short if the particle size is large. For example, the dropping rate of the HDI with a diameter of $2 \mathrm{~mm}$ can quickly reach a magnitude of meters per second in the melt. Therefore, these inclusions are apt to remain in the ingot and eventually become defects in the VAR melting process. However, in the EBCHM process, these inclusions will segment to the shell and be removed permanently. From the aspect of economics, the cost of titanium sponge and alloying elements account for about $40 \%$ to $60 \%$ of the total cost of the ingot. One of the important routes to lowering the cost is using titanium wastes. However, the using of machining wastes and leftovers will increase not only the risk of nitrides and oxides in the titanium, but also the risk of hard machine tool debris. As the EBCHM technology needs not to prepare an electrode and can use titanium wastes as raw materials, it becomes a suitable way of titanium wastes recycling.

\section{Migrating of inclusions in EBCHM process}

The EBCHM has a heating source with very high energy, long residual time of melt, and a long flow path. The LDI can be removed by resolving or decomposing, while the HDI can be eliminated by sedimentation. These features make the EBCHM the most effective technology of inclusions removal. Han et al. ${ }^{[43]}$ studied the removal of LDI and HDI of TC4 alloy in the EBCHM process by adding synthesized inclusions (nitride sponge titanium particles and WC particles). Zhang et al. ${ }^{[44]}$ researched the resolving of nitride inclusions in the EBCHM process of TC4 alloy by adding TiN inclusions in the feeding port. These experiment results all show that the EBCHM has a superior effect of inclusion removal.

Bellot et al. ${ }^{[45]}$ studied the migrating behavior of inclusions in the electron beam melting of a nickel-based alloy button. The results show that the factors affecting migration are mainly natural convection and Marangoni force. Natural convection influences the migration of inclusions in the internal melt while Marangoni flow influences the migration on the melt surface. Marangoni flow is intrinsically caused by surface tension. So, the flow is affected by the composition and temperature of the melt. For example, the researchers observed the inversion of the Marangoni effect due to the presence of sulphur. Marangoni

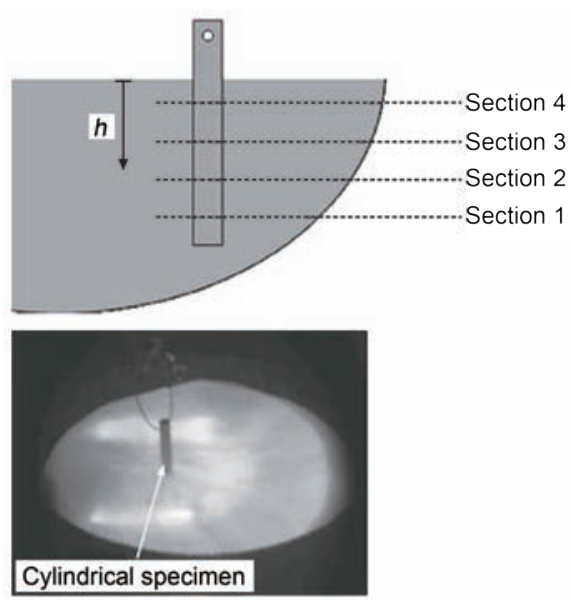

Fig. 8: Resolving experiment of tungsten bar $^{[42]}$
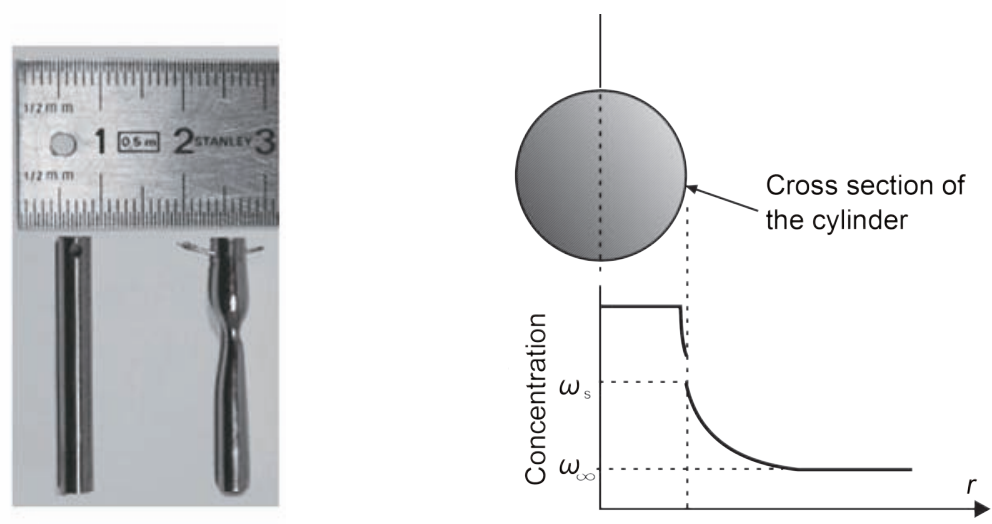

Fig. 9: Resolving model of HDI ${ }^{[42]}$ 
force drives inclusions to move under the electron beam when the concentration of sulphur is higher than critical value. As these areas have very high temperature, the result is that the inclusions may be burned. When the concentration of sulphur is lower than the critical value, inclusions are concentrated to low temperature zones of the surface. The simulation results show that the movement of inclusions greatly depends on their size. Particles larger than $100 \mu \mathrm{m}$ are affected mainly by buoyancy while particles with the size of several microns are mainly affected by the turbulence. Funagane researched the migrating behavior of LDI in the cold hearth and verified by experiments ${ }^{[46]}$. The results show that the surface tension gradient will cause strong flow, which drives melt flow from high temperature zone to low temperature zone. In this experiment, the strong Marangoni flow resulted by electron beam heating was used to control the melt flow in the cold hearth. The simulation results show that LDI will be constrained in the cold hearth, as shown in Fig. 10.
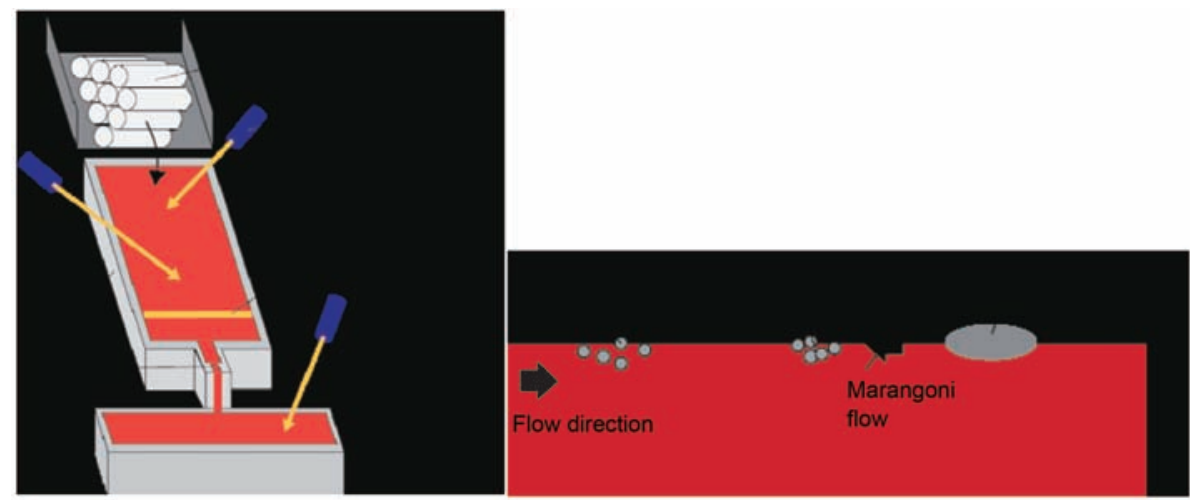

Fig. 10: EBCHM process (left) and migration of inclusions driven by Marangoni force (right) ${ }^{[46]}$

Bellot et al. ${ }^{[47]}$ simulated the EBCHM melting process of titanium alloy. Their work considered the momentum, mass transfer and heat transfer. Two models were used to calculate the trajectories of particles. One was used to describe the resolving of particles and another describes the melt flow. The resolving model of inclusions is shown in Fig. 6. From this model, the nitrogen distribution and the size of different phases can be calculated. A particle was introduced from the hearth inlet to initiate the trajectory. The trajectory was achieved by solving basic dynamic equations and with the help of Language method. The effects of gravity, buoyancy and forces of fluid (drag force and inertia force) were considered. The density of particles is constant, and the initial velocity and accelerated velocity were set as zero. The effect of initial size $(250,300$ and $500 \mu \mathrm{m})$ of particles on the trajectories was researched.

The trajectories of inclusions with different densities are shown in Fig. 11. It indicates that inclusions with neutral density move along the cold hearth, while heavy particles almost instantly sediment to the melt bottom. When particle density is lower than that of the melt, particles will ascend to the melt surface and be transmitted to the shell. After colliding with the shell, particles may have two different behaviors. One possibility is that the particle returns to the internal melt again. Sequential collisions will greatly increase the residual time of particles in the melt, which increases the possibility of removal by resolving. Another is that when particles collide with the shell, a boundary layer forms on the particle surface by the diffusion of nitrogen. Particles are pinned on the shell and can be removed. Figure 12 shows that particle size has a great influence on the resolving time. Particles with the radius of 300 and $200 \mu \mathrm{m}$ were completely removed, while particles with the radius of $500 \mu \mathrm{m}$

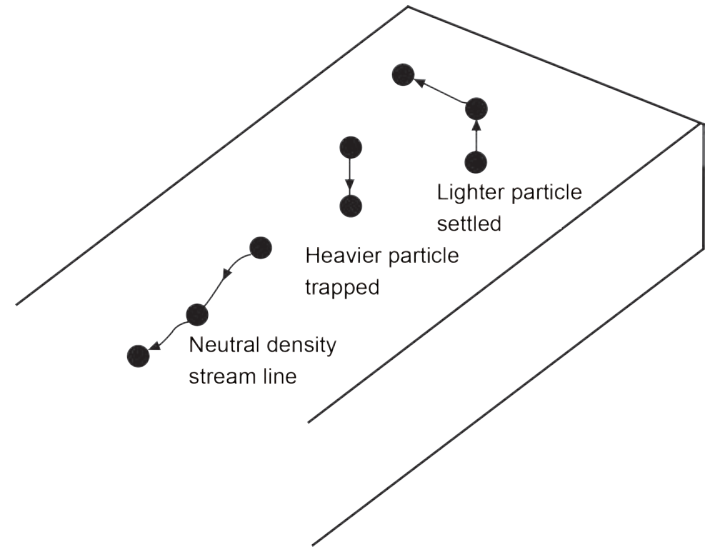

Fig. 11: Trajectories of inclusions with different densities ${ }^{[47]}$

can flow through the cold hearth. The trajectories at different melting rates were calculated. The residual time of particles was reduced with the increase of melting rate, and particles with the radius of $300 \mu \mathrm{m}$ can flow through the cold hearth.

According to the above analysis, sedimentation and resolving are the main mechanisms of inclusion removal in the EBCHM process. Medium density inclusions can be removed by resolving, which is related to particle size and residual time. In the EBCHM process, the residual time of particles can be increased by lowering the melting rate, but this will increase the evaporation of elements. Therefore, these two contradictory factors need to be considered in the production practice.

\section{Summary}

High and low-density particles are two main inclusions in the 
melting process of titanium and titanium alloys. This article comprehensively introduces the possible sources, characteristics, removing mechanisms, related experiment researches and physical models of these inclusions.

Low density inclusions mainly come from nitrogen and oxygen contamination of titanium. These inclusions are difficult to detect and are the most harmful defects of titanium. With the increase of nitrogen, $\beta, \alpha$ and $\delta$ phases form respectively. The resolving of a low density inclusion is a reaction diffusion process accompanying solid phase transformation. The diffusion of nitrogen is very slow. This is the major obstacle of inclusion removal. In the EBCHM process, the size of LDI has a great influence on the resolving time. For some big particles, it cannot be completely resolved. These particles will eventually flow into the crystallizer.

High density inclusions mainly come from refractory metal of the raw material and are relatively easy to detect. The resolving rate of these inclusions is very slow. Traditional melting methods, like VAR, cannot remove HDI effectively. While in the EBCHM process, HDI will be completely removed by dropping.

The common melting methods of titanium and titanium alloy are specified. Compared with other melting methods, EBCHM has the best capability of removing high and low-density inclusions for the following reasons: the high melting temperature is a benefit for the resolving and burning of inclusions; the vacuum degree of the chamber is high, so the melt won't react with air; the flow path of the melt is long, so inclusions have enough residual time to resolve; the shell can capture the inclusions; the flow caused by surface tension is conducive to the removal of inclusions. In production practice, besides the removing of inclusions, the evaporation and homogeneity also need to be considered before choosing appropriate melting methods.
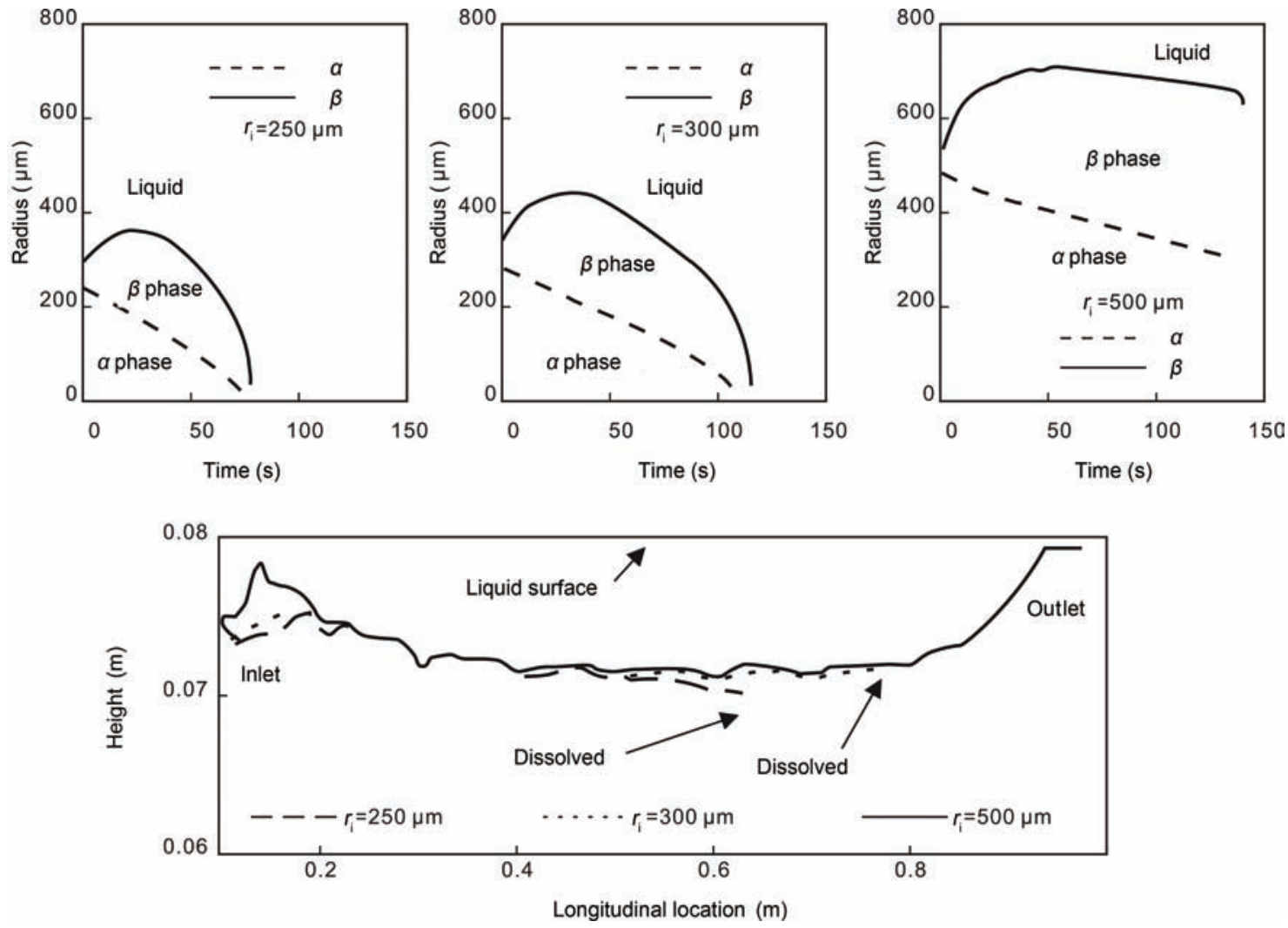

Fig. 12: Resolving process of inclusions of different sizes ${ }^{[47]}$

\section{References}

[1] Luo Lei, Yang Guanjun, Mao Xiaonan, et al. Development of electron beam cold hearth melting technology for titanium alloys. Casting Forging Welding, 2009, 38(19): 56-59. (In Chinese)

[2] Semiatin S L, Kobryn P A, Ivasishin O M, et al. The role of modeling in the development of advanced processes for metallic aerospace alloys. Metals and Materials International, 2004, 10(6): 589-603.

[3] Jin Hexi, Wei Kexiang, Li Jianmin, et al. Research development of titanium alloy in aerospace industry. The Chinese Journal of Nonferrous Metals, 2015, 25 (2): 280-292. (In Chinese)

[4] Yuan Guosen, Yan Lipeng, Han Yanyan, et al. Application progress of titanium alloy. Hot Working Technology, 2017,46 (4): 13-16. (In Chinese)

[5] Immarigeon J P, Holt R T, Koul A K, et al. Light materials for aircraft applications. Materials Characterization, 1995, 35: 4167.

[6] Brewer W D, Bird R K, Wallance T A. Titanium alloys processing for high speed aircraft. Materials Science and Engineering $A$, 1998, 243: 299-304.

[7] Yamada M. An overview on the development of titanium alloys for non-aerospace application in Japan. Materials Science and Engineering A, 1996, 213: 8-15.

[8] Cai Jianmin, Li Zhenxi, Ma Jimin, et al. Research and development of $600{ }^{\circ} \mathrm{C}$ high temperature titanium alloy for 
aeroengine. Materials Review, 2005,19 (1): 50-53. (In Chinese)

[9] Zhang Yingming, Zhou Lian, Sun Jun, et al. Research development of electron beam cold hearth remelting of $\mathrm{Ti}$ alloys. Titanium Industry Progress, 2008, 25 (4): 14-19. (In Chinese)

[10] Lei Wenguang, Zhao Yongqing, Han Dong, et al. Development of melting technology for titanium and titanium alloys. Materials Review, 2016, 30(3): 101-106. (In Chinese)

[11] Suzuki K. An introduction to the extraction, melting and casting technologies of titanium alloys. Metals and Materials International, 2001, 7(6): 587-604.

[12] Shu Qun, GuoYongliang, Chen Ziyong, et al. Development of casting and melting technology of titanium alloy. Materials Science \& Technology, 2004,12(3): 332-336. (In Chinese)

[13] Wang Gao, Li Xianjun, Wen Zhigang, et al. Production technology for low cost titanium products. Review, 2013, 3: 8-12. (In Chinese)

[14] Gao Ting, Zhao Liang, Ma Baofei, et al. Present situation and development trend of titanium alloy casting technology. Hot Working Technology, 2014, 43(21): 5-11. (In Chinese)

[15] Chen Xianming. Development of melting and casting technology of titanium alloy. Journal of Zhaoging University, 2010, 31(2): 2025. (In Chinese)

[16] Troshchenko V T, Pokrovskii V V, Rarusevich V L, et al. Effect of inclusion contaminants on the crack resistance of ductile titanium alloys. Strength of Materials, 1991(8): 654-862.

[17] Cai Jianming, Ma Jimin, Hao Mengyi, et al. Hard alpha defect in titanium alloys and its control using plasma arc cold hearth melting technique. Failure Analysis and Prevention, 2007, 2(2): 51-57. (In Chinese)

[18] Li Yuxian, Yang Lichun. Electron beam cold hearth melting technology and discussion. Metal World, 2012(6): 51-55. (In Chinese)

[19] Liu Qianli, Li Xiangming, Jiang Yehua. Research progress of electron beam cold hearth melting for titanium and titanium alloys. Hot Working Technology, 2016,45(9): 9-14. (In Chinese)

[20] Tian Shifan, Ma Jimin. Development and applications of electron beam cold hearth melting. Journal of Materials Engineering, 2012(2): 77-85. (In Chinese)

[21] Qin Guihong, Wang Wanbo, Ji Bo, et al. Introduction and application of industrial cooling hearth melting technology. The Chinese Journal of Nonferrous Metals, 2010, 20(s1): 877-880. (In Chinese)

[22] Chen Feng, Chen Li, Guo Bin, et al. Advantages and disadvantages of electron beam cold hearth melting. The Chinese Journal of Nonferrous Metals, 2010(s1): 873-876. (In Chinese)

[23] Yu Lanlan, Mao Xiaonan, Zhang Yingming, et al. Development of electron-beam cold hearth single melt process for titanium alloy ingots. Titanium Industry Progress, 2009, 26(2): 14-18. (In Chinese)

[24] Duan Junwei. Cold hearth technology and applications for titanium and alloys. Nonferrous Metals Process, 2011, 40(1): 4142. (In Chinese)

[25] Ma Rongbao, Chen Feng, Guo Bin. Development of EBCHR and discussion of its remelting process. Titanium Industry Progress, 2008, 25 (5): 37-40. (In Chinese)

[26] Wood J R. Producing Ti-6Al-4V plate from single melt EBCHM ingot. Journal of Metals, 2002(2): 56-58.

[27] Huang Haiguang, Cao Zhanyuan, Li Zhimin, et al. Electron beam cold hearth melting process research of titanium recycled material. 2015, 44(7): 137-144. (In Chinese)

[28] Zhao Jiong, Yang Guoqing, Qiao Lu, et al. Study on EB+VAR melting process of TC11 ingot for important usage. China Titanium Industry, 2015(3): 31-34. (In Chinese)

[29] Bao Shujuan. Research development on electron beam cold hearth melting of TC4 alloy. Development and Application of Materials, 2012(5): 87-90. (In Chinese)

[30] Li Xiong, Pang Kechang, Guo Hua, et al. Melting technology of wrought $\mathrm{Ti}$ and $\mathrm{Ti}$ alloy. The Chinese Journal of Nonferrous Metals, 2010, 20 (s1): 906-913. (In Chinese)

[31] Mitchell A. The electron beam melting and refining of titanium alloys. Materials Science and Engineering A, 1999, 263: 217223.

[32] Shen Haijun, Wang Ning, Zhang Ge. Hard a inclusions in titanium and titanium alloy and their removal methods. Shanghai Metals, 2010, 32(2): 38-45. (In Chinese)

[33] Henry J L, Hill S D, Schaller J L, et al. Nitride inclusions in titanium ingots: a study of possible sources in the production of Magnesium-reduced sponge. Metallurgical Transactions, 1973, 4: 1859-1864.

[34] Bewlay B P, Gigliotti M F X. Dissolution rate measurements of TiN in Ti-6242. Acta Mater., 1997(1): 357-370.

[35] Wang D J, Mitchell A M. Effects of alloying elements on nitrogen diffusion behavior around TiN/Ti interface aregion in as-cast titanium alloys. Trans. Nonferrous Met. Soc. China, 2001, 5: 738-742.

[36] Bellot J P, Foster B, Hans S. Dissolution of hard-alpha inclusions in liquid titanium alloys. Metallurgical and Materials Transactions B, 1996, 28: 1001-1010.

[37] Wang Hongquan, Yun Pengfei, Liu Huan, et al. Analysis on sources of tungsten inclusion during melting titanium alloy ingot in vacuum consumable arc furnace and its counter measures. Special Steel Technology, 2017, 23(1): 43-47. (In Chinese)

[38] Mitchell A. Melting, casting and forging problems in titanium alloys. Materials Science and Engineering A, 1998, 243: 257262.

[39] Mitchell A. Melting, casting and forging problems in titanium alloys. Journal of Metals, 1997(6): 40-43.

[40] Yamanaka A, Ichihashi. Dissolution of refractory elements to titanium alloy in VAR. ISIJ International, 1992, 32: 600-606.

[41] Li Xuefei, Huang Lijun, Huang $\mathrm{Xu}$, et al. Analysis of hard impurity defects of titanium alloy. Aerospace Materials \& Technology, 2015, 6: 74-77. (In Chinese)

[42] Ghazal G, Jardy A, Chapelle P, et al. On the dissolution of nitrided titanium defects during vacuum arc remelting of $\mathrm{Ti}$ alloy. Metallurgical and Materials Transactions B, 2010, 41B: 646-659.

[43] Han Mingchen, Zhang Yingming, Zhou Yigang, et al. Elimination of LDI and HDI in TC4 alloy during electron beam cold melting, Rare Metal Materials and Engineering, 2008, 37(4): 665-669. (In Chinese)

[44] Zhang Yingming, Zhou Lian, Sun Jun, et al. An investigation on electron beam cold hearth melting of Ti64 alloy. Rare Metal Materials and Engineering, 2008, 37(11): 1973-1977.

[45] Bellot J P, Defay B, Jourdan J, et al. Inclusion behavior during the electron beam button melting test. Journal of Materials Engineering and Performance, 2011, 21(10): 2140-2146.

[46] Funagane $\mathrm{H}$. Electron beam melting toward inclusionfree titanium alloys. In: 9th International Symposium on Electromagnetic Processing of Materials (EPM2018), Awaji Yumebutai, 2018: 1-4.

[47] Bellot J P, Hess E, Abletzer D. Aluminum volatilization and inclusion removal in the electron beam cold hearth melting of $\mathrm{Ti}$ alloys. Metallurgical and Materials Transactions B, 2000 (31B): 845-854. 